representativeness - sentinel sites may not be representative of the entire pregnant population.

Consequently new methods for antenatal HIV surveillance need investigation. Since the inception of the SA programme to prevent HIV transmission from mother to child (PMTCT), more than 95\% of the healthcare system integrates PMTCT HIV testing into routine antenatal care. ${ }^{[3,4]}$ Furthermore, the district health information system (DHIS), which gathers aggregate data from each facility in each district, has been updated to include routine data elements on HIV testing and HIV test results in general and among pregnant women. Data on second tests during pregnancy have also recently been added to the DHIS.

A growing consensus has posited that, in the context of antiretroviral therapy, PMTCT expansion and DHIS strengthening, alternative surveillance methods and data sources are increasingly available and should be explored to address the concerns associated with UAT-based ANCHSS. ${ }^{[2]}$ The World Health Organization developed guidelines for countries to evaluate the utility of routine data from PMTCT programmes 'for HIV sentinel surveillance among pregnant women' as outlined above. ${ }^{[2]}$ These guidelines recognise the denominator and double counting problems with DHIS data, which require rectification. ${ }^{[5]}$

This then begs the following questions: $(i)$ should time and energy be invested in studying SA DHIS data and comparing these with SA ANCHSS data? (ii) should the quality of routine antenatal HIV testing procedures be assessed? and (iii) should SA ANCHSS be stopped?

We answer 'yes' to the first two questions, as strong routine monitoring systems are critical for management and planning. In addressing the third question, we believe that if routine data are used to monitor antenatal HIV prevalence, intermittent, periodic ANCHSS may still be needed to corroborate results. However, ANCHSS should be changed to linked, named testing to circumvent duplication and ethical issues. As our previous national work has shown that $>95 \%$ of mothers accept named testing, uptake of antenatal HIV testing would not be significantly reduced by named, linked testing. ${ }^{[6]}$

Acknowledgement. This work was supported by funds from the South African Medical Research Council.

Palesa Nkomo

Medical Research Council Health Systems Research Unit, Pretoria, South Africa palesa.nkomo@mrc.ac.za

\title{
Tracking antenatal HIV prevalence in South Africa
}

To the Editor: South Africa (SA) has been conducting annual crosssectional anonymous unlinked antenatal HIV seroprevalence surveys in sentinel sites (ANCHSS) for more than 20 years. ${ }^{[1]}$ These sites are randomly selected using probability proportional to size sampling (PPS) methods as this combines a random approach with a bias towards larger clinics, resulting in a self-weighted sample. ${ }^{[1]}$ Firsttime antenatal attendees are enrolled into the ANCHSS, and blood for unlinked anonymous antenatal HIV testing (UAT) is drawn at the same time as first antenatal booking bloods.

Without refuting the usefulness of ANCHSS for tracking the antenatal HIV epidemic, five main concerns have recently been raised: ${ }^{[2]}$ (i) high cost of ANCHSS implementation; (ii) high cost of duplicate HIV testing - every pregnant woman is tested anonymously for HIV infection using laboratory enzyme-linked immunoassay tests and is also routinely tested for HIV infection using clinic-based rapid tests; (iii) timing - the survey mainly measures HIV prevalence once during pregnancy; (iv) ethical anonymous results are not returned to pregnant women; and $(v)$

\author{
Ameena Goga \\ Medical Research Council Health Systems Research Unit, Pretoria, South Africa, and \\ Department of Paediatrics, University of Pretoria \\ 1. National Department of Health, South Africa. The 2012 National Antenatal Sentinel HIV \& Herpes \\ Simplex Type-2 Prevalence Survey in South Africa. http://www.health-e.org.za/wp-content/ \\ Simplex Type-2 Prevalence Survey in South Africa. http://www.health-e.or
uploads/2014/05/ASHIVHerp_Report2014_22May2014.pdf (accessed 8 April 2015). \\ 2. World Health Organization. Guidelines for Assessing the Utility of Data from Prevention of Mother- \\ . World Health Organization. Guidelines for Assessing the Utility of Data from Prevention of Mother-
To-Child Transmission (PMTCT) Programmes for HIV Sentinel Surveillance Among Pregnant Women. \\ http://apps.who.int/iris/bitstream/10665/85512/1/9789241505611_eng.pdf (accessed 8 April 2015). \\ 3. Department of Health, South Africa. National Consolidated Guidelines for the Prevention of Mother- \\ To-Child Transmission of HIV (PMTCT) and the Management of HIV in Children, Adolescents \\ and Adults. http://www.sahivsoc.org/upload/documents/HIV\%20guidelines\%20_Jan\%202015.pdf \\ (accessed 8 April 2015). \\ 4. Woldesenbet S, Goga A, Jackson D, et al. Mother-To-Child Transmission of HIV (PMTCT). Evaluation \\ of the Early Infant Diagnosis Service in Primary Health Care Facilities in South Africa: Report on \\ Results of Situational Assessment. http://www.mrc.ac.za/healthsystems/SituationalAssessment2012. \\ pdf (accessed 8 April 2015) \\ 5. Health Systems Trust. Health Systems Barometer, 2013/2014. http://www.health-e.org.za/wp-content/ \\ uploads/2014/10/DHB_2013-14.pdf (accessed 8 April 2015) \\ 6. Goga AE, Dinh TH, Jackson DJ for the SAPMTCTE study group. Early (4-8 Weeks Post-delivery) \\ 6oga AE, Dinh TH, Jackson DJ for the SAPMTCTE study group. Early (4-8 Weeks Post-delivery)
Population-level Effectiveness of WHO PMTCT Option A, South Africa, 2011. South African Medical \\ Population-level Effectiveness of WHO PMTCT Option A, South Africa, 2011. South African Medical
Research Council, National Department of Health of South Africa and PEPFAR/US Centers for Disease \\ Research Council, National Department of Health of South Africa and PEPFAR/US Centers for Disease
Control and Prevention. 2013. http://www.mrc.ac.za/healthysstems/SAPMTCTE2011.pdf (accessed 8 \\ April 2015).
}

S Afr Med J 2015;105(4):329. DOI:10.7196/SAMJ.9472 